\title{
A Method to Establish the Dynamic Models of Multibody System Based on Kane's Equations
}

\author{
Yangwei Zhong \\ School of Energy \& Power Engineering, NJUST, \\ Nanjing, China \\ zyw_601@163.com
}

\author{
Liangming Wang \\ School of Energy \& Power Engineering, NJUST, \\ Nanjing, China \\ lmwang802@163.com
}

\begin{abstract}
According to the tree structure description of the multibody system, the Kane's equations to establish the dynamic equations of the system are proposed. The forms of the equations are general and simple. Moreover, the equations are not only minimum in dimension but also convenient for computer calculating. The parachute-submunition system is taken as the modelling object which includes submunition, plate, parachute. The dynamic equations of the three parts are established respectively through which the dynamic and kinematics equations for the whole system are established. The influences of the drag coefficient and the crosswind velocity on the scan parameters are simulated by computer programming. The simulation results are in accordance with the flight principle and exterior ballistic theory, which verifies the reasonableness of the modelling method and the established dynamic equations.
\end{abstract}

Keywords-Dynamic model; Kane's equation; Parachutesubmunition system; Simulation.

\section{INTRODUCTION}

How to establish a mathematical model which is both general and convenient for computer calculating is the research emphasis on the dynamics of multibody system. Various methods are used to establish the dynamic equations of multibody system such as Newton-Euler's method, Lagrange's method and Kane's method. However, NewtonEuler's method needs to calculate a lot of internal forces and constraint forces. That make it complicated to establish the model if the system is complex. Lagrange's method needs to calculate the dynamic functions and their derivatives that will increase large amounts of calculations. Relative studies have shown that Kane's method has more advantages on the modelling of multibody system.

Kane's method is an analytical method of multibody system that is proposed and developed by Kane T.R. in the 1960s [1]. Kane's method selects the general velocities to describe the motion of both holonomic and nonholonomic system. The established equations do not require computation of workless constraint forces and moments, which makes this method easily to be programmed and more computationally efficient compared with the other methods.

Kane's method caused a lot of attention since it was put forward and researchers discussed its characters in many papers. Desloge and Keat discussed the relationship between Kane's and Appell's equations [2,3]. London summarized and compared the Newton's, Lagrange's, D'alembert's and Kane's equations [4]. Townsend studied the equivalence of Kane's, Appell's and Lagrange's equation [5]. Kane summarized the four characteristics of the Kane's equations and compared the advantages with other methods in application [6, 7].

The terminal sensitive submunition is a kind of smart ammunition that applied advanced sensing instrument technology and EFP warhead technology [8] Researches in the dynamics models of parachute-submunition system are found based on Lagrange Mechanics [9], Kane method [10], and Newton method [11].

This paper begins with the tree structure description of the multibody system followed by the Kane's equations for multibody system are proposed. With this method, the paper calculates the equations of the submunition, plate, parachute and finally gets the dynamic equations of the parachutesubmunition system. At last, the corresponding numerical analyses and conclusions are given.

\section{KanE’s Method OF Multibody System}

\section{A. Structure Description of the System}

As the system shown in Fig. 1, body 1 is the reference body of all the other bodies, which is called the main body. For the two connected bodies, the body close to the main body is called the higher body and the other is called the lower body.

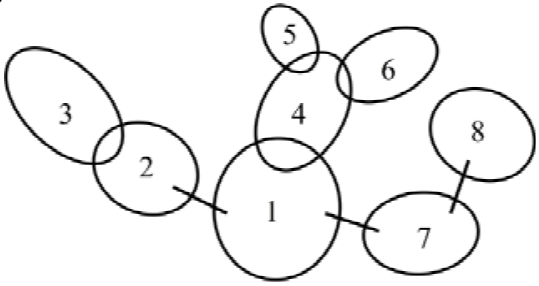

Figure 1. Tree structure description of the system

In order to establish the dynamic equations of the system, we can separate the system into independent parts and establish the dynamic equations of each part respectively. Then we can combine the established dynamic equations of each part into the dynamic equations of the system based on the connected relationship of each part.

According to Kane's equations, after generalized velocities $u_{p}$ of the body having been determined, the 
absolute velocity $\boldsymbol{v}$ and absolute angular velocity $\boldsymbol{\omega}$ of any point on the body can be expressed as the linear superposition of partial velocity $\boldsymbol{v}_{p}$ and partial angular velocity $\omega_{p}[1]$

$$
\boldsymbol{v}=\sum_{p=1}^{n} u_{p} \boldsymbol{v}_{p}, \boldsymbol{\omega}=\sum_{p=1}^{n} u_{p} \boldsymbol{\omega}_{p}
$$

Where, $p$ is the number of generalized velocities of the body. Therefore, the partial velocity and partial angular velocity reflect the motion features of the whole system as well as each body. So the motion features of the system can be obtained from the partial velocity and partial angular velocity of each body.

\section{B. Motion Description of the System}

As shown in Fig. 2, $i$ and $j$ are two adjacent bodies of a multibody system and $i$ is the higher body. The coordinate variables that describe the motion of the body $j$ relative to the body $i$ are selected as the generalized velocities. $h_{i}$ and $b_{i j}$ are two points on the body $i . h_{i}$ is the reference point that describes the motion of the body $i$ and $b_{i j}$ is the reference point that describes the motion of the body $j$ relative to the body $i . h_{j}$ is a reference point on the body $j$ at which establishs two coordinate systems: coordinate system $h_{j i}$ that moves with the body $i$ and coordinate system $h_{j}$ that moves with the body $j$.

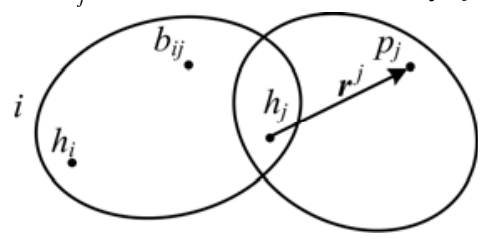

Figure 2. Motion description of the system

The motion of the body $j$ relative to the body $i$ can be described by the translation of the point $h_{j}$ relative to the point $b_{i j}$ and the rotation of the coordinate system $h_{j}$ relative to the coordinate system $h_{j i}$.Suppose that the body $i$ has belongs to the system A whose generalized velocities are $u_{p_{0}}$, where $p_{0}$ is from 1 to $n^{A}$. The body $j$ connects with the body $i$ and the degree-of-freedom (DOF) of the body $j$ relative to body $i$ is $n_{j i}$. Therefore the DOF of the body $j$ is

$$
n_{j}=n^{A}+n^{j i}
$$

According to the expansion of the generalized velocities, the partial velocity and partial angular velocity of any point $p_{j}$ on the body $j$ can be expressed as

$$
\begin{array}{r}
\boldsymbol{v}^{j}=\sum_{p=1}^{n_{j}} u_{p} \boldsymbol{v}_{p}^{j}, \boldsymbol{v}_{p}^{j}=\boldsymbol{v}_{p}^{h_{j}}+\boldsymbol{\omega}_{p}^{r_{j}} \times \boldsymbol{r}^{j}, \\
\boldsymbol{\omega}^{j}=\sum_{p=1}^{n_{j}} u_{p} \boldsymbol{\omega}_{p}^{j}, \boldsymbol{\omega}_{p}^{j}=\boldsymbol{\omega}_{p}^{i}+\boldsymbol{\omega}_{p}^{(j, i)}
\end{array}
$$

Where, $\boldsymbol{v}_{p}^{h_{j}}$ is the partial velocity of the point $h_{j} ; \boldsymbol{\omega}_{p}^{r_{j}}$ is the partial angular velocity of the rotation of the body $j$; $\boldsymbol{\omega}_{p}^{i}$ is the partial angular velocity of the body $i, \boldsymbol{\omega}_{p}^{(j, i)}$ is the partial angular velocity of the rotation of the body $j$ relative to the body $i$.

\section{Dynamic Models of the System}

Suppose that the body $j$ belongs to the system $\mathrm{B}$, then the Kane's equations of the system $C$ according to the systems A and B are as follows.

- Generalized inertial force of each system

The generalized inertial force of each system is

$$
f_{p}^{*(k)}=-\sum_{p, q=1}^{n}\left(m_{p q}^{(k)} \dot{u}_{q}+c_{p q}^{(k)} u_{q}\right)
$$

Where

$$
\begin{gathered}
m_{p q}^{(k)}=m^{(k)} \boldsymbol{v}_{p}^{(k)} \cdot \boldsymbol{v}_{q}^{(k)}+\boldsymbol{\omega}_{p}^{(k)} \cdot\left(\boldsymbol{I}^{(k)} \cdot \boldsymbol{\omega}_{q}^{(k)}\right), \\
c_{p q}^{(k)}=m^{(k)} \boldsymbol{v}_{p}^{(k)} \cdot \dot{\boldsymbol{v}}_{q}^{(k)}+\boldsymbol{\omega}_{p}^{(k)} \cdot\left[\boldsymbol{I}^{(k)} \dot{\boldsymbol{\omega}}_{q}^{(k)}+\boldsymbol{\omega}^{(k)} \times\left(\boldsymbol{I}^{k} \cdot \boldsymbol{\omega}_{q}^{(k)}\right)\right], \\
k=\mathrm{A}, \mathrm{B}
\end{gathered}
$$

- $\quad$ Generalized active force of each system

The generalized active force of each system is

$$
f_{p}^{(k)}=\boldsymbol{v}_{p}^{(k)} \cdot \boldsymbol{F}^{(k)}+\boldsymbol{\omega}_{p}^{(k)} \cdot \boldsymbol{M}^{(k)}
$$

Where $\boldsymbol{F}^{(k)}$ and $\boldsymbol{M}^{(k)}$ are the resultant force and resultant moment that do work on system $k$.

- Dynamic models of the whole system

According to the Kane's equations, the sum of the generalized inertial force and the generalized a force is zero, and the dynamic equations of the system are

$$
\left[m_{p q}\right]\left[\dot{u}_{q}\right]+\left[c_{p q}\right]\left[u_{q}\right]=\left[f_{p}\right]
$$

Where

$$
\left[m_{p q}\right]=\sum_{k}\left[m_{p q}^{(k)}\right],\left[c_{p q}\right]=\sum_{k}\left[c_{p q}^{(k)}\right],\left[f_{p}\right]=\sum_{k}\left[f_{p}^{(k)}\right]
$$




\section{DyNAMIC MODEL OF THE PARACHUTE-SUBMUNITION} SYSTEM

\section{A. Basic Hypothesis}

- The parachute and its additional parts are a rigid body that have a certain shape and mass.

- The parachute-submunition system consist of three rigid bodies: submunition, plate and parachute. The submunition moves with six DOFs which is as the main body. The plate connects with the submunition by the column hinge and it rotates relatively with one DOF. The parachute connect with the plate by the virtual spherical hinge and it rotates relatively with three DOFs. Therefore the total DOFs of the multibody system is ten. The structure of the parachute-submunition system is as the Fig. 3 follow.

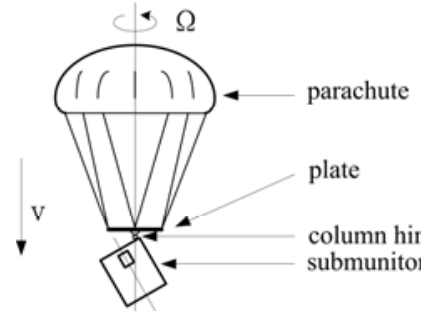

Figure 3. Structure of the parachute-submunition system

\section{B. Coordinate Systems}

- The ground coordinate system, oxyz. The origin $o$ is located in the center of the muzzle; The axis $o x$ is the intersecting line of shooting plane and horizontal plane. The axis oy is in the vertical plane and its positive direction is upward; The axis $O z$ is determined by the right-hand rule.

- The translation coordinate system, $c^{(1)} x y z$ : The origin $c^{(1)}$ is located in the centroid of the submunition. The translation coordinate system is obtained by the translation of the oxyz .

- The submunition coordinate system, $c^{(1)} x_{1}^{(1)} y_{1}^{(1)} z_{1}^{(1)}$ : The axis $c^{(1)} x_{1}^{(1)}$ is in accordance with the submunition axis and the positive direction is pointing to the flight direction. The axis $c^{(1)} y_{1}^{(1)}$ and $c^{(1)} z_{1}^{(1)}$ are in the cross section of the submunition. The submunition coordinate system rotates with the submunition.

- The submunition velocity coordinate system, $c^{(1)} x_{2}^{(1)} y_{2}^{(1)} z_{2}^{(1)}$. The axis $c^{(1)} x_{2}^{(1)}$ is in accordance with the submunition velocity. The axis $c^{(1)} y_{2}^{(1)}$ is in the vertical plane that is perpendicular to the axis $c^{(1)} x_{2}^{(1)}$.Its positive direction is upward; The axis $c^{(1)} z_{2}^{(1)}$ is determined by the right-hand rule.
- The submunition axis coordinate system, $c^{(1)} \xi^{(1)} \eta^{(1)} \varsigma^{(1)}$. The axis $c^{(1)} \xi^{(1)}$ is in accordance with the submunition axis. The axis $c^{(1)} \eta^{(1)}$ and $c^{(1)} \varsigma^{(1)}$ are in the cross section of the submunition. The submunition axis coordinate system doesn't rotate with the submunition.

- $\quad$ The plate coordinate system, $c^{(2)} x_{1}^{(2)} y_{1}^{(2)} z_{1}^{(2)}$. The origin $c^{(2)}$ is located in the centroid of the plate. The axis $c^{(2)} X_{1}^{(2)}$ is in accordance with the plate axis and the positive direction is pointing to the flight direction. The axis $c^{(2)} y_{1}^{(2)}$ and $c^{(2)} z_{1}^{(2)}$ are in the cross section of the plate. The plate coordinate system rotates with the plate.

- The parachute coordinate system, $c^{(3)} x_{1}^{(3)} y_{1}^{(3)} z_{1}^{(3)}$. The origin $c^{(3)}$ is located in the centroid of the parachute. The axis $c^{(3)} x_{1}^{(3)}$ is in accordance with the parachute axis and the positive direction is pointing to the flight direction. The axis $c^{(3)} y_{1}^{(3)}$ and $c^{(3)} z_{1}^{(3)}$ are in the cross section of the parachute. The parachute coordinate system rotates with the parachute.

- The parachute velocity coordinate system, $c^{(3)} x_{2}^{(3)} y_{2}^{(3)} z_{2}^{(3)}$. The axis $c^{(3)} x_{2}^{(3)}$ is in accordance with the parachute velocity. The axis $c^{(3)} y_{2}^{(3)}$ is in the vertical plane that is perpendicular to the axis $c^{(3)} x_{2}^{(3)}$.Its positive direction is upward; The axis $c^{(3)} z_{2}^{(3)}$ is determined by the right-hand rule.

- The parachute axis coordinate system, $c^{(3)} \xi^{(3)} \eta^{(3)} \varsigma^{(3)}$. The axis $c^{(3)} \xi^{(3)}$ is in accordance with the parachute axis. The axis $c^{(3)} \eta^{(3)}$ and $c^{(3)} \varsigma^{(3)}$ are in the cross section of the parachute. The parachute axis coordinate system doesn't rotate with the parachute.

\section{Motion Description of Parachute-Submunition System}

- Motion description of the submunition The position vector of the submunition's centroid is

$$
\boldsymbol{r}_{c}^{(1)}=x \boldsymbol{i}+y \boldsymbol{j}+z \boldsymbol{k}
$$

The velocity vector of the submunition's centroid is

$$
\boldsymbol{v}_{c}^{(1)}=\dot{\boldsymbol{r}}_{c}^{(1)}=\dot{x} \mathbf{i}+\dot{y} \boldsymbol{j}+\dot{z} \boldsymbol{k}
$$

The angular velocity vector of the submunition is

$$
\boldsymbol{\omega}^{(1)}=\omega_{x} \boldsymbol{i}_{x_{1}}+\omega_{y} \boldsymbol{j}_{y_{1}}+\omega_{z} \boldsymbol{k}_{z_{1}}
$$

The generalized velocities are selected as

$$
\left[u_{p}\right]=\left[\begin{array}{lllllll}
\dot{x} & \dot{y} & \dot{z} & \omega_{x} & \omega_{y} & \omega_{z} & \cdots
\end{array}\right]
$$


According to the generalized velocities, the velocity and angular velocity vector of the submunition are expressed as

$$
\boldsymbol{v}_{c}^{(1)}=\sum_{p=1}^{10} u_{p} \boldsymbol{v}_{p}^{(1)}, \boldsymbol{\omega}^{(1)}=\sum_{p=1}^{10} u_{p} \boldsymbol{\omega}_{p}^{(1)}
$$

The partial velocity vectors $\boldsymbol{v}_{p}^{(1)}$, partial angular velocity vectors $\boldsymbol{\omega}_{p}^{(1)}$ and their derivatives are shown in the following Tab.1. In order to calculate conveniently, the partial velocity vector and partial angular velocity vector are resolved in the parallel motion coordinate system and body coordinate system respectively.

- Motion description of the plate

$\mathrm{O}_{2}$ is selected as a reference point on the plate and it is the center of the column hinge. Since the plate is thin, assuming that the center of column hinge $\mathrm{O}_{2}$ and the centroid of plate $c^{(2)}$ are coincident. The plate revolves around $\mathrm{O}_{2}$ with one degree of freedom.

The position vector of $O_{2}$ is

$$
\boldsymbol{r}_{o_{2}}^{(2)}=\boldsymbol{r}_{c}^{(2)}=\boldsymbol{r}_{c}^{(1)}+\boldsymbol{r}^{(2,1)}
$$

Where $\boldsymbol{r}^{(2,1)}$ is the radius vector from the centroid of the submunition to the center of the column hinge. It is expressed as component forms in the submunition coordinate system.

The angular velocity vector of the plate is

$$
\omega^{(2)}=\omega^{(1)}+\omega^{(2,1)}
$$

Where $\boldsymbol{\omega}^{(2,1)}$ is the relative angular velocity vector between the plate and the submunition. It is expressed as follows.

$$
\boldsymbol{\omega}^{(2,1)}=\omega_{z}^{(2,1)} \boldsymbol{k}_{z_{1}}^{(2)}
$$

The generalized velocities are selected as

$$
\left[u_{p}\right]=\left[\begin{array}{llllllll}
\dot{x} & \dot{y} & \dot{z} & \omega_{x} & \omega_{y} & \omega_{z} & \omega_{z}^{(2,1)} & \cdots
\end{array}\right]
$$

The velocity vector of $c^{(2)}$ is

$$
\boldsymbol{V}_{c}^{(2)}=\dot{\boldsymbol{r}}_{c}^{(2)}=\dot{\boldsymbol{r}}_{c}^{(1)}+\boldsymbol{\omega}^{(1)} \times \boldsymbol{r}^{(2,1)}
$$

According to the generalized velocities, the velocity and angular velocity vector of the plate are expressed as follows.

$$
\boldsymbol{v}_{c}^{(2)}=\sum_{p=1}^{10} u_{p} \boldsymbol{v}_{p}^{(2)}, \boldsymbol{v}_{p}^{(2)}=\boldsymbol{v}_{p}^{(1)}+\vec{\omega}_{p}^{(1)} \times \boldsymbol{r}^{(2,1)},
$$

$$
\boldsymbol{\omega}^{(2)}=\sum_{p=1}^{10} u_{p} \omega_{p}^{(2)}, \omega_{p}^{(2)}=\omega_{p}^{(1)}+\omega_{p}^{(2,1)}
$$

The derivatives of partial velocity vector $\boldsymbol{v}_{p}^{(2)}$ and partial angular velocity vector $\boldsymbol{\omega}_{p}^{(2)}$ with respect to time are expressed as follows.

$$
\begin{gathered}
\dot{\boldsymbol{v}}_{p}^{(2)}=\dot{\boldsymbol{v}}_{p}^{(1)}+\dot{\boldsymbol{\omega}}_{p}^{(1)} \times \boldsymbol{r}^{(2,1)}+\boldsymbol{\omega}_{p}^{(1)} \times\left(\boldsymbol{\omega}^{(1)} \times \boldsymbol{r}^{(2,1)}\right), \\
\dot{\boldsymbol{\omega}}_{p}^{(2)}=\dot{\boldsymbol{\omega}}_{p}^{(1)}+\dot{\boldsymbol{\omega}}_{p}^{(2,1)}
\end{gathered}
$$

Where the relative partial angular velocity vectors $\omega_{p}^{(2,1)}$ and their derivatives $\dot{\boldsymbol{\omega}}_{p}^{(2,1)}$ are shown in the Tab. 2.

TABLE I. PARTIAL VELOCITY VECTORS, PARTIAL ANGULAR VELOCITY VECTORS AND THEIR DERIVATIVES

\begin{tabular}{|c|c|c|c|c|}
\hline \multirow{2}{*}{ subscript $p$} & \multicolumn{5}{|c|}{ partial velocity vectors, partial angular velocity vectors and } \\
\cline { 2 - 5 } & $\boldsymbol{v}_{p}^{(1)}$ & $\boldsymbol{\omega}_{p}^{(1)}$ & $\dot{\boldsymbol{v}}_{p}^{(1)}$ & $\dot{\boldsymbol{\omega}}_{p}^{(1)}$ \\
\hline 1 & $\boldsymbol{i}$ & $\boldsymbol{0}$ & $\boldsymbol{0}$ & $\boldsymbol{0}$ \\
\hline 2 & $\boldsymbol{j}$ & $\boldsymbol{0}$ & $\boldsymbol{0}$ & $\boldsymbol{0}$ \\
\hline 3 & $\boldsymbol{k}$ & $\boldsymbol{0}$ & $\boldsymbol{0}$ & $\boldsymbol{0}$ \\
\hline 4 & $\boldsymbol{0}$ & $\boldsymbol{i}_{x_{1}}$ & $\boldsymbol{0}$ & $\boldsymbol{\omega}^{(1)} \times \boldsymbol{i}_{\chi_{1}}$ \\
\hline 5 & $\boldsymbol{0}$ & $\boldsymbol{j}_{y_{1}}$ & $\boldsymbol{0}$ & $\boldsymbol{\omega}^{(1)} \times \boldsymbol{j}_{y_{1}}$ \\
\hline 6 & $\boldsymbol{0}$ & $\boldsymbol{k}_{z_{1}}$ & $\boldsymbol{0}$ & $\boldsymbol{\omega}^{(1)} \times \boldsymbol{k}_{z_{1}}$ \\
\hline $7 \ldots 10$ & $\boldsymbol{0}$ & $\boldsymbol{0}$ & $\boldsymbol{0}$ & $\boldsymbol{0}$ \\
\hline
\end{tabular}

TABLE II. RELATIVE PARTIAL ANGULAR VELOCITY VECTORS AND THEIR DERIVATIVES

\begin{tabular}{|c|c|c|c|}
\hline \multirow{2}{*}{$\begin{array}{c}\text { relative partial angular velocity } \\
\text { vectors and their derivatives }\end{array}$} & \multicolumn{3}{|c|}{ subscript $p$} \\
\cline { 2 - 4 } & $1 \ldots 6$ & 7 & $8 \ldots 10$ \\
\hline $\boldsymbol{\omega}_{p}^{(2,1)}$ & $\mathbf{0}$ & $\boldsymbol{k}_{\mathrm{z}_{1}}^{(2)}$ & $\mathbf{0}$ \\
\hline$\dot{\boldsymbol{\omega}}_{p}^{(2,1)}$ & $\mathbf{0}$ & $\boldsymbol{\omega}^{(2)} \times \boldsymbol{k}_{\mathrm{z}_{1}}^{(2)}$ & $\mathbf{0}$ \\
\hline
\end{tabular}

- Motion description of the parachute

$O_{3}$ is taken as the reference point on the parachute that coincides with $\mathrm{O}_{2}$ and the parachute revolves around $\mathrm{O}_{3}$ with three degrees of freedom.

The position vector of the parachute's centroid is

$$
\boldsymbol{r}_{c}^{(3)}=\boldsymbol{r}_{O_{3}}+\boldsymbol{r}_{c}^{(3)}
$$

Where $\boldsymbol{r}_{c}^{(3)}$ is the radius vector from the centroid of the plate to the centroid of the parachute and it is expressed as component forms in the plate coordinate system.

The angular velocity vector of the parachute is 


$$
\boldsymbol{\omega}^{(3)}=\boldsymbol{\omega}^{(2)}+\boldsymbol{\omega}^{(3,2)}
$$

Where $\boldsymbol{\omega}^{(3,2)}$ is the relative angular velocity vector between the parachute and the plate that is expressed as

$$
\boldsymbol{\omega}^{(3,2)}=\omega_{x}^{(3,2)} \boldsymbol{i}_{x_{1}}^{(3)}+\omega_{y}^{(3,2)} \boldsymbol{j}_{y_{1}}^{(3)}+\omega_{z}^{(3,2)} \boldsymbol{k}_{z_{1}}^{(3)}
$$

The generalized velocities are selected as

$$
\left[u_{p}\right]=\left[\begin{array}{llllllllll}
\dot{x} & \dot{y} & \dot{z} & \omega_{x} & \omega_{y} & \omega_{z} & \omega_{z}^{(2,1)} & \omega_{x}^{(3,2)} & \omega_{y}^{(3,2)} & \omega_{z}^{(3,2)}
\end{array}\right]
$$

The velocity vector of $c^{(3)}$ is

$$
\boldsymbol{v}_{c}^{(3)}=\dot{\boldsymbol{r}}_{o_{3}}+\boldsymbol{\omega}^{(3)} \times \boldsymbol{r}_{c}^{(3)}=\boldsymbol{v}_{o_{2}}+\boldsymbol{\omega}^{(3)} \times \boldsymbol{r}_{c}^{(3)}
$$

According to the generalized velocities, the velocity and angular velocity vectors of the parachute are expressed as follows.

$$
\begin{gathered}
\boldsymbol{v}_{c}^{(3)}=\sum_{p=1}^{10} u_{p} \boldsymbol{v}_{p}^{(3)}, \boldsymbol{v}_{p}^{(3)}=\boldsymbol{v}_{p}^{(2)}+\boldsymbol{\omega}_{p}^{(3)} \times \boldsymbol{r}_{c}^{(3)}, \\
\boldsymbol{\omega}^{(3)}=\sum_{p=1}^{10} u_{p} \boldsymbol{\omega}_{p}^{(3)}, \boldsymbol{\omega}_{p}^{(3)}=\boldsymbol{\omega}_{p}^{(2)}+\boldsymbol{\omega}_{p}^{(3,2)}
\end{gathered}
$$

The derivatives of the partial velocity vectors $\boldsymbol{v}_{p}^{(3)}$ and the partial angular velocity vectors $\omega_{p}^{(3)}$ with respect to time are expressed as follows.

$$
\begin{gathered}
\dot{\boldsymbol{v}}_{p}^{(3)}=\dot{\boldsymbol{v}}_{p}^{(2)}+\dot{\boldsymbol{\omega}}_{p}^{(3)} \times \boldsymbol{r}_{c}^{(3)}+\boldsymbol{\omega}_{p}^{(3)} \times\left(\boldsymbol{\omega}^{(3)} \times \boldsymbol{r}_{c}^{(3)}\right) \\
\dot{\boldsymbol{\omega}}_{p}^{(3)}=\dot{\boldsymbol{\omega}}_{p}^{(2)}+\dot{\boldsymbol{\omega}}_{p}^{(3,2)}
\end{gathered}
$$

The relative partial angular velocity vectors $\boldsymbol{\omega}_{p}^{(3,2)}$ and their derivatives are listed in the table below.

TABLE III. RELATIVE PARTIAL ANGULAR VELOCITY VECTORS AND THEIR DERIVATIVES

\begin{tabular}{|c|c|c|c|c|}
\hline $\begin{array}{c}\text { relative partial angular } \\
\text { velocity } \\
\text { vectors and their } \\
\text { derivatives }\end{array}$ & \multicolumn{4}{|c|}{ subscript $p$} \\
\cline { 2 - 5 } & $1 \ldots 7$ & 8 & 9 & 10 \\
\hline $\boldsymbol{\omega}_{p}^{(3,2)}$ & $\mathbf{0}$ & $\boldsymbol{i}_{x_{1}}^{(3)}$ & $\boldsymbol{j}_{y_{1}}^{(3)}$ & $\boldsymbol{k}_{z_{1}}^{(3)}$ \\
\hline$\dot{\boldsymbol{\omega}}_{p}^{(3,2)}$ & $\mathbf{0}$ & $\boldsymbol{\omega}^{(3)} \times \mathbf{i}_{x_{1}}^{(3)}$ & $\boldsymbol{\omega}^{(3)} \times \boldsymbol{j}_{y_{1}}^{(3)}$ & $\boldsymbol{\omega}^{(3)} \times \boldsymbol{k}_{\mathrm{z}_{1}}^{(3)}$ \\
\hline
\end{tabular}

\section{DYNAMIC MODELS OF THE SYSTEM}

\section{A. Generalized Inertial Force of the System}

The generalized inertial force of each body is

$$
f_{p}^{*(k)}=-\sum_{p=1}^{n}\left(m_{p q}^{(k)} \dot{u}_{q}+c_{p q}^{(k)} u_{q}\right)
$$

Where

$$
\begin{gathered}
m_{p q}^{(k)}=m^{(k)} \boldsymbol{v}_{p}^{(k)} \cdot \boldsymbol{v}_{q}^{(k)}+\boldsymbol{\omega}_{p}^{(k)} \cdot \boldsymbol{I}^{(k)} \cdot \boldsymbol{\omega}_{q}^{(k)}, \\
c_{p q}^{(k)}=m^{(k)} \boldsymbol{v}_{p}^{(k)} \cdot \dot{\boldsymbol{v}}_{q}^{(k)}+\boldsymbol{\omega}_{p}^{(k)} \cdot\left(\boldsymbol{I}^{(k)} \dot{\boldsymbol{\omega}}_{q}^{(k)}+\boldsymbol{\omega}^{(k)} \times \boldsymbol{I}^{k} \cdot \boldsymbol{\omega}_{q}^{(k)}\right),
\end{gathered}
$$

$$
k=1,2,3
$$

B. Generalized Active Force of the System

The generalized active force of each body is

$$
f_{p}^{(k)}=\boldsymbol{v}_{p}^{(k)} \cdot \boldsymbol{F}^{(k)}+\boldsymbol{\omega}_{p}^{(k)} \cdot \boldsymbol{M}^{(k)}
$$

Where $\boldsymbol{F}^{(k)}$ and $\boldsymbol{M}^{(k)}$ are resultant force and resultant moment that do work on the body $k$ respectively.

\section{Dynamic Equations of the System}

The dynamic models of the system are

$$
\left[m_{p q}\right]\left[\dot{u}_{q}\right]+\left[c_{p q}\right]\left[u_{q}\right]=\left[f_{p}\right]
$$

Where

$$
\left[m_{p q}\right]=\sum_{k=1}^{3}\left[m_{p q}^{(k)}\right],\left[c_{p q}\right]=\sum_{k=1}^{3}\left[c_{p q}^{(k)}\right],\left[f_{p}\right]=\sum_{k=1}^{3}\left[f_{p}^{(k)}\right]
$$

\section{SIMULATION}

The scan speed of the system mainly depend on the total mass of the system and the drag coefficient of the parachute. When the drag coefficients are taken as $0.7,1.17,1.5,2$, respectively, the curves of the scan parameters are shown as the figures follows.

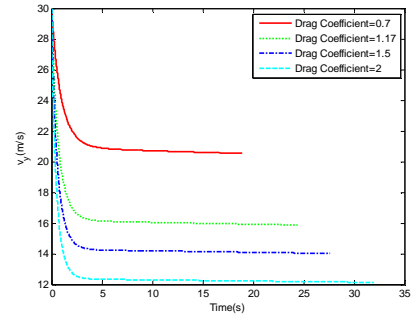

Figure 4. Scan speed curves in different drag coefficients

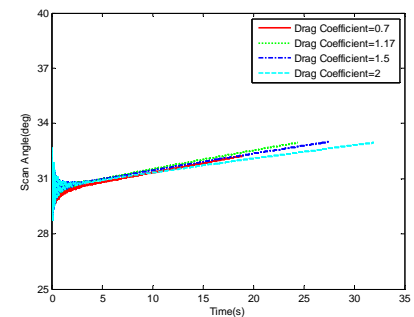

Figure 5. Scan angle curves in different drag coefficients 


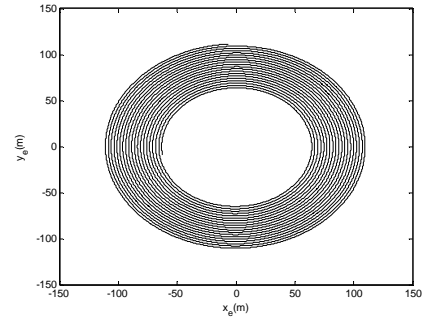

Figure 6. Stable scan trajectory curve when drag coefficients is 0.7

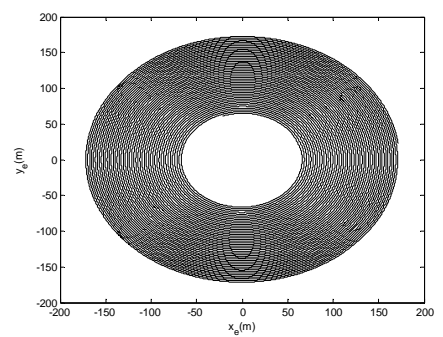

Figure 7. Scan trajectory curve when drag coefficients is 1.5

The Figures show that, with the increase of the drag coefficients, the scan speed of the system decreases. As a result, the scan time increases and the scan trajectory becomes intensive. The scan angles are stabilized around $32^{\circ}$, that indicate that the drag coefficient has little effect on the scan angle.

\section{CONCLUSION}

The paper provides an applied method for solving the problem of dynamic models of the multibody system. This method is based on the Kane's equations and the structure and motion descriptions of the system. Taking the parachutesubmuniton system as an object, the structure and motion descriptions of are introduced. And the dynamic equations of the whole system are established. Combining with some concrete examples, the results of the scan parameters are calculated. The simulation results are in accordance with the flight principle and exterior ballistic theory, which verify the reasonableness of the established dynamic model.

\section{REFERENCES}

[1] Kane T R, and Levinson DA, “Dynamics: theory and applications”, McGraw Hill, 1985.

[2] Desloge, E. A. "Relationship between Kane's equations and the Gibbs-Appell equations", Journal of Guidance, Control, and Dynamics, Vol. 1, No. 10, 1987.

[3] Keat, J, “Comment on relationship between Kane's equations and the Gibbs-Appell equations". Journal of Guidance, Control, and Dynamics, Vol. 6, No. 10, 1987.

[4] London, K. W, "Derivation of dynamic equations and evaluation of the Kane methodology”. AIAA/AAS Astrodynamics Conference[C], Portland, 1990.

[5] Miles.A.Townsend, “Equivalence of Kane's, Gibbs-Appell's, and Lagrange's equations". Journal of Guidance, Control, and Dynamics, Vol. 5, No. 15, 1992, pp. 1289-1292.

[6] Kane T R, "A new method to establish dynamic equations". Advances in Mechanics, Vol. 2, No. 13, 1983, pp. 257-262.

[7] Kane T R, Levinson DA, "Formulation of equations of motion for complex spacecraft”. Journal of Guidance and Control, Vol. 2, No. 3, 1978.

[8] Rui Guo, Rongzhong Liu, Zhengjun Shi, "Particle-rigid body dynamics model for terminal-sensitive submunition”, World Journal of Modelling and Simulation, Vol. 1, No. 2, 2005, pp. 105-110.

[9] Yong Zhu, Li Liu, “Dynamic Model of parachute-projectile systems based on Lagrange mechanics", ACTA AERONAU TICA ET ASTRONAU TICA SINICA, Vol. 7, No. 30, 2009, pp. 1208-1213.

[10] Yonggao Xie, Zizeng Qin, "Decelerating and stable scanning modeling of terminal munition by Kane method", AEROSPACE SHANGHAI, Vol. 1, No. 1, 2003, pp. 17-20.

[11] Jingrong Shu, et al, "Analysis on three body motion of parachuteprojectile systems”, ACTA AERONAU TICA ET ASTRONAU TICA SINICA, Vol. 6, No. 22, 2001, pp. 481-485. 